\title{
Microvascular disorders induced by malaria infected red blood cells: a computational mechanical study using the biological particle method
}

\author{
T. Yamaguchi ${ }^{1}$, H. Kondo ${ }^{2}$, Y. Imai ${ }^{2} \&$ T. Ishikawa ${ }^{2}$ \\ ${ }^{1}$ Department of Biomedical Engineering, \\ Graduate School of Biomedical Engineering, Tohoku University, Sendai, \\ Japan \\ ${ }^{2}$ Department of Bioengineering and Robotics, \\ Graduate School of Engineering, Tohoku University, Sendai, Japan
}

\begin{abstract}
We simulated malarial microvascular blood flow disturbances by using a new particle method of biological solid-fluid interaction analysis especially developed for the analysis of malaria infection. Particle based spatial discretization and the sub time step time integration could provide us with stable computations for micro scale blood flow involving interaction with many cells. We performed numerical simulation of the stretching of infected red blood cells and the results agreed well with experimental results. Our model successfully simulated the flow of infected red blood cells into narrow channels.
\end{abstract}

Keywords: malaria, computational fluid dynamics, particle method, red blood cell, microcirculation, mechanical properties of cell membrane.

\section{Introduction}

Malaria is one of the most serious infectious diseases on earth. There are 500 million patients with 2 million deaths arising from malaria infection. When a parasite invades and matures inside a red blood cell (RBC), the infected RBC (IRBC) becomes stiffer and cytoadherent. These changes are postulated to link to microvascular blockage [1]. Several researchers have investigated cell mechanics of IRBCs using recent experimental techniques. Methods to quantify 
the stiffness of the IRBCs include the micropipette aspiration [2-4] and optical tweezers [5]. Suresh et al. [5] used optical tweezers to investigate the change of mechanical response of IRBCs at different development stages. They clarified that the shear modules can increase by ten-fold in the final schizont stage. Microfluidics has also been used to investigate the pathology of malaria. Shelby et al. [9] investigated the effect of IRBCs on the capillary obstruction and demonstrated that IRBCs in the late stages of the infection cannot pass through micro channels that have diameters smaller than those of the IRBCs. These studies are reviewed in [6-8].

These experimental studies are still limited to the effect of the single infected cell. However, microvascular blockage may be a hemodynamics problem, involving the interactions between IRBCs, healthy RBCs and endothelial cells. This is due to the limitation of the current experimental techniques. Firstly, it is still difficult to observe the RBC behaviour interacting with many other cells, even with the recent confocal microscope. Secondly, three-dimensional information on the flow field is hard to obtain. Thirdly, capillaries in the human body are circular channels with complex geometry, but such complex channels cannot be created in micro scale. Instead, numerical modelling can be a strong tool for further understanding the pathology of malaria. Finite element modelling was performed for the stretching of an IRBC by optical tweezers [5]. Dupin et al. [10] also modelled the stretching of the IRBC using the lattice Boltzmann method. We have also developed a hemodynamic model involving adhesive interactions [11]. In this report, we present our methodology and preliminary numerical results.

\section{Method}

\subsection{Particle method}

Blood is a suspension of RBCs, white blood cells, and platelets in plasma. An RBC consists of cytoplasm enclosed by a thin membrane. Assuming that plasma and cytoplasm are incompressible and Newtonian fluids, the governing equations are described as

$$
\begin{gathered}
\frac{D \rho}{D t}=0, \\
\frac{D \mathbf{u}}{D t}=-\frac{1}{\rho} \nabla p+\nu \nabla^{2} \mathbf{u}+\mathbf{f},
\end{gathered}
$$

where the notation $t$ refers to the time, $\rho$ is the density, $\mathbf{u}$ is the velocity vector, $\mathrm{p}$ is the pressure, $v$ is the dynamic viscosity, $\mathbf{f}$ is the external force per unit mass, and $D / D t$ is the Lagrangian derivative.

Our model is based on a particle method. All the components of blood are represented by particles (Fig. 1). Note that each particle is not a real fluid particle but a discrete point for computation. Fluid variables are calculated at the computational point and it is moved by the calculated advection velocity every time step. In conventional mesh methods, each computational point requires the 
connection to neighbouring points for the discretization of Eqs. (2) and (3) and thus the computational meshes are generated. When a red blood cell approaches another other cell, however, the computational meshes can be distorted and destroyed easily. In contrast to mesh methods, the particle method does not require such computational meshes and the computation is stable even when many cells are interacting with each other. Another advantage of the particle method is the coupling with the front-tracking. The particle method tracks the front of the membrane using the membrane particles and the no-slip condition on the membrane is directly imposed to Eq. (3) by using the position and velocity of membrane particles. We use the moving particle semi-implicit (MPS) method [12] for solving Eqs. (2) and (3). In the MPS method, differential operators in the governing equations are approximated using the weight function, for example,

$$
\begin{aligned}
& \nabla \phi_{i}=\frac{d}{n^{0}} \sum_{j \neq i} \frac{\phi_{j}-\phi_{i}}{\left|\mathbf{r}_{i j}\right|^{2}} \mathbf{r}_{i j} w\left(\mid \mathbf{r}_{i j}\right), \\
& \nabla^{2} \phi_{i}=\frac{2 d}{\lambda n^{0}} \sum_{j \neq i}\left(\phi_{j}-\phi_{i}\right) w\left(\left|\mathbf{r}_{i j}\right|\right),
\end{aligned}
$$

where $\phi$ is a fluid variable, $d$ is the space dimension number, $\mathrm{n}^{0}$ is the reference particle number density, $\lambda$ is the constant, $\mathbf{r}$ is the position of particle, $\mathbf{r}_{i j}=\mathbf{r}_{j}-\mathbf{r}_{\mathbf{i}}$, and $w$ is the weight function.

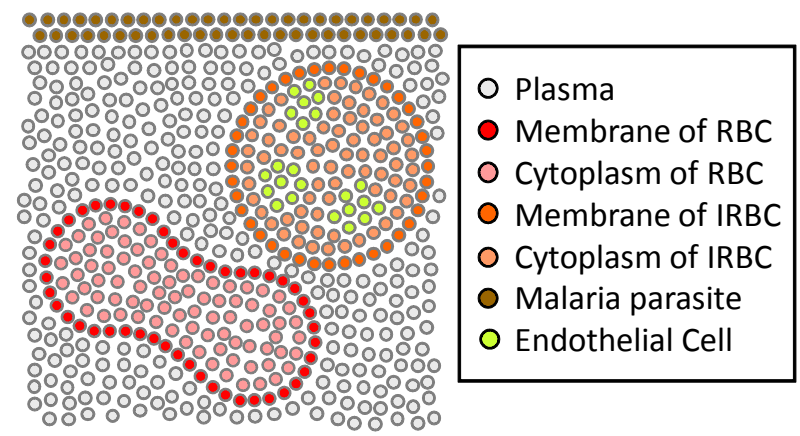

Figure 1: $\quad$ Scheme of free mesh particle model of blood. All the components of blood are represented by the finite number of particles (computational points). Velocity and pressure are calculated at the position of each particle and it is moved by the calculated advection velocity every time step.

\subsection{IRBC model}

The membrane of IRBCs is represented by the two-dimensional network consisting of the finite number of particles (Fig. 2). Particle $j$ is connected to particle $i$ by linear spring, giving a force, 


$$
\mathbf{F}_{j}^{s}=k_{s}\left(\left|\mathbf{r}_{i j}\right|-l_{0}\right) \frac{\mathbf{r}_{i j}}{\left|\mathbf{r}_{i j}\right|},
$$

where $k_{s}$ is the spring constant and $l_{0}$ is the equilibrium distance. A bending force is also considered in our model:

$$
\begin{aligned}
& \mathbf{F}_{j}^{b}=\mathbf{F}_{k}^{b}=\frac{\mathbf{F}_{i}^{b}+\mathbf{F}_{l}^{b}}{2}, \\
& \mathbf{F}_{i}^{b}=k_{b} \tan \left(\frac{\theta}{2}\right) \mathbf{n}_{i j k}, \\
& \mathbf{F}_{l}^{b}=k_{b} \tan \left(\frac{\theta}{2}\right) \mathbf{n}_{j k l},
\end{aligned}
$$

where $k_{b}$ is the spring constant, $\theta$ is the angle between the triangles $\Delta_{i j k}$ and $\Delta_{j k l}$, and $\mathbf{n}_{i j k}$ is the normal vector to the triangle $\Delta_{i j k}$. The external force per mass is given as

$$
\mathbf{f}_{i}=\frac{\mathbf{F}_{j}^{s}+\mathbf{F}_{j}^{b}}{\rho V_{0}},
$$

where $\mathrm{V}_{0}$ is the reference volume $\mathrm{V}_{0}=\mathrm{r}_{0}{ }^{3}$ and $\mathrm{r}_{0}$ is the averaged particle distance at the initial time step. Note that the spring constants $k_{s}$ and $k_{b}$ should be model parameters to control the deformation of IRBCs. As described in the next section, we adjust these model parameters through numerical experiments.

A malaria parasite inside IRBCs is modelled by a rigid object constructed by some particles. The treatment of the rigid object in the MPS method was proposed by Koshizuka et al. [13].

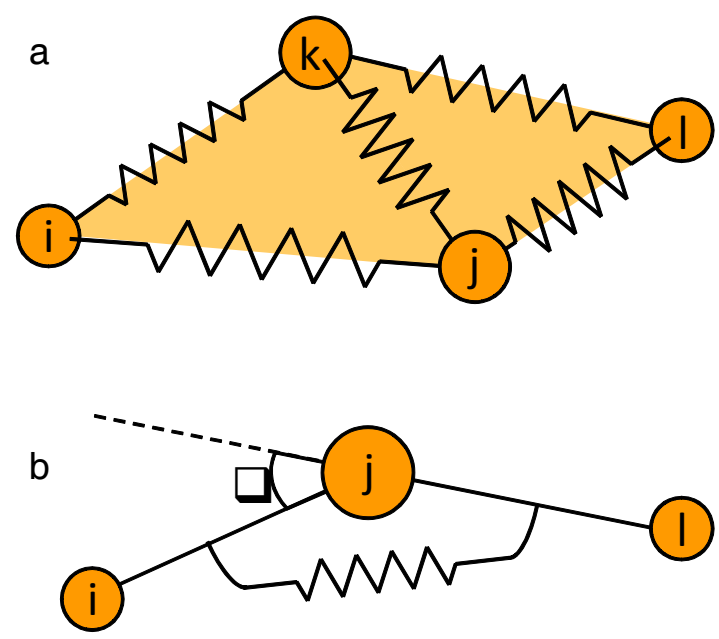

Figure 2: $\quad$ Spring network model of IRBC membrane. (a) Each membrane particle is connected to the neighbouring membrane particles by a spring with linear elasticity. (b) To express the deformation of the thin membrane, the bending force is also introduced. 


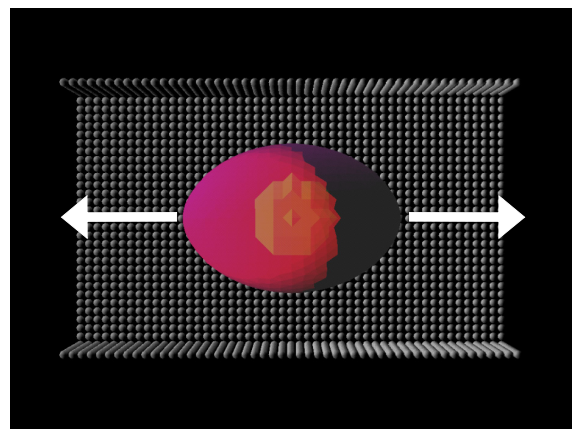

Figure 3: Stretching of IRBCs. An IRBC is replaced at the centre of the computational domain at the initial time step. The IRBC is stretched horizontally by a constant force. Governing equations are time integrated until the steady state solution is obtained.

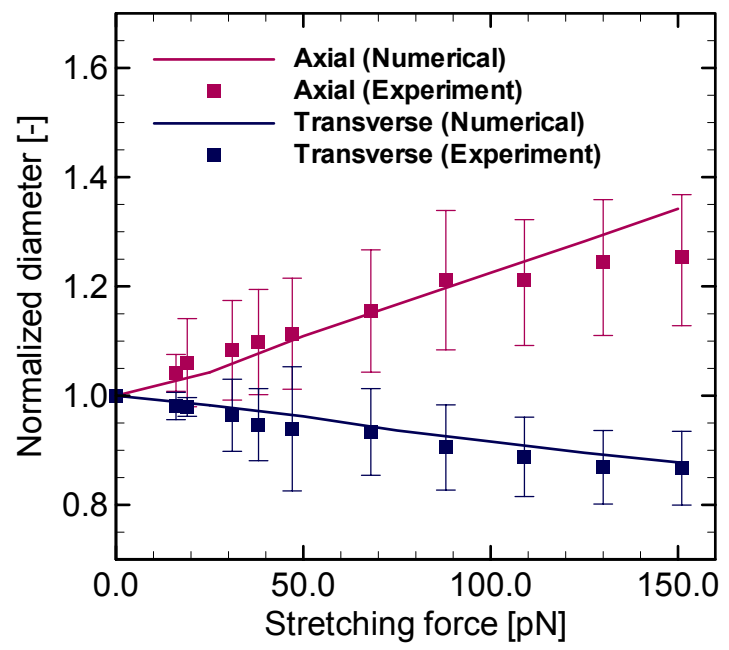

Figure 4: Comparison between the numerical results and the experimental results [11] for stretching of IRBCs in the schizont stage. Symbols indicate the experimental results and lines are the numerical results. The upper line represents the results for axial diameter and the lower line represents that for transverse diameter. The numerical results agree well with the experimental ones.

\section{Results}

Suresh et al. [5] performed stretching of IRBCs by optical tweezers to quantify the mechanical response of IRBCs. They measured the axial and transverse diameters of IRBCs at different development stages with several stretching 
forces. We examine this stretching test numerically to validate our model. In the three-dimensional computational domain, we put an IRBC at initial time step. Then we stretch the IRBC horizontally with a stretching force as shown in Fig. 3. The governing equations are time-integrated until the axial and transverse diameters are well converged. If the obtained results do not follow the experimental results, the values of model parameters $\mathrm{ks}$ and $\mathrm{kb}$ are changed and the computation is carried out again. Finally we can get the appropriate values of the model parameters. Final results of IRBCs in the schizont stage are presented in Fig. 4. The numerical results agree well with the experimental results by Suresh et al. [5].

\section{Discussion}

We have proposed a numerical model of three-dimensional hemodynamics arising from malaria infection. A particle based spatial discretization and sub time step time integration method are employed to stably simulate many cell interactions in micro scale blood flow.

To validate our model, we examined stretching of IRBCs. When we used appropriate values for the spring coefficients $\mathrm{ks}$ and $\mathrm{kb}$, the numerical results agreed well with the experimental results. Small differences can be found in the axial diameter for high stretching force. It may be improved using a non-linear spring model for membrane. However, compared with previously presented numerical models, such as a finite element model [5] and a Lattice Boltzmann model [10], our model has similar accuracy for the stretching of IRBCs.

Our model also successfully simulated flow into narrow channels, mimicking microcirculation in human body. We tested two sizes of narrow channels: a 6mm-square channel and a 4-mm-square channel. Both HRBCs and IRBCs in the schizont stage passed through a 6-mm-square channel. While HRBCs also flowed into the 4-mm-square channel, schizont IRBCs occluded the flow. These results follow the experimental observation by Shelby et al [9], where they revealed that IRBCs in the late trophozoite and schizont stages occluded flow into the channel with $4 \mathrm{~mm}$ width.

\section{Conclusion}

In this report, we proposed a numerical model of three-dimensional hemodynamics arising from malaria infection. Particle based spatial discretization and the sub time step time integration can provide us stable computations for the micro scale blood flow involving the interaction with many cells. We performed the stretching of IRBCs and the numerical results agreed well with experimental results. Our model successfully simulated flow of IRBCs into narrow channels. Here, we have not considered the adhesion property of IRBCs. We have already developed an adhesion model [9] and this adhesion model can be applied easily to the current hemodynamic model. 


\section{References}

[1] Cooke BM, Mohandas N, and Coppell RL. The malaria-infected red blood cell: structural and functional changes. Advances in Parasitology 26, 1-86, 2001.

[2] Nash GB, O'Brien E, Goldon-Smith EC, and Dormandy JA. Abnormalities in the mechanical properties of red blood cells caused by plasmodium falciparum. Blood 74, 855-861, 1989.

[3] Paulitschke M, and Nash GB. Membrane rigidity of red blood cells parasitized by different strains of Plasmodium falciparum. $J$ Lab Clin Med 122, 581-589, 1993.

[4] Lim CT, Zhou EH, and Quek ST. Mechanical models for living cells-a review. J Biomech 39, 195-216, 2006.

[5] Suresh S, Spatz J, Mills JP, Micoulet A, Dao M, Lim CT, Beil M, and Seufferlein T. Connection between single-cell biomechanics and human disease states: gastrointestinal cancer and malaria. Acta Biomater 1, 15-30, 2005.

[6] Lim CT, Zhou EH, Li A, Vedula SRK, and Fu HX. Experimental techniques for single cell and single molecule biomechanics. Mater Sci Eng C 26, 1278-1288, 2006.

[7] Lim CT. Single cell mechanics study of the human disease malaria. $J$. Biomech Sci Eng 1, 82-92, 2006.

[8] Lee GYH, and Lim CT. Biomechanics approaches to studying human diseases. Trends Biotech 25, 112-118, 2007.

[9] Shelby JP, White J, Ganesan K, Rathod PK, and Chiu DT. A microfluidic model for single-cell capillary obstruction by Plasmodium falciparuminfected erythrocytes. PNAS 100, 14618-14622, 2003.

[10] Dupin MM, Halliday I, Care CM, and Munn LL. Lattice Boltzmann modelling of blood cell dynamics. Int J CFD 22, 481-492, 2008.

[11] Kondo $\mathrm{H}$, Imai $\mathrm{Y}$, Ishikawa $\mathrm{T}$, Tsubota $\mathrm{K}$, and Yamaguchi $\mathrm{T}$. Hemodynamic analysis of microcirculation in malaria infection. Ann Biomed Eng, accepted.

[12] Koshizuka S, and Oka Y, Moving-particle semi-implicit method for fragmentation of incompressible fluid. Nucl Sci Eng 123, 421-434, 1996.

[13] Koshizuka S, Nobe A, and Oka Y. Numerical analysis of breaking waves using the moving particle semi-implicit method. Int $J$ numer meth Fluids 26, 751-769, 1998. 